INPLASY

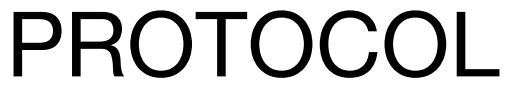

To cite: Chen et al. A metaanalysis of high mobility group 1 frame protein levels in cerebrospinal fluid and serum of patients with epilepsy. Inplasy protocol 2021120029. doi:

10.37766/inplasy2021.12.0029

Received: 04 December 2021

Published: 04 December 2021

Corresponding author:

Yue Chen

329930608@qq.com

Author Affiliation:

Yangtze River Shipping

General Hospital.

Support: Hospital Research Fund.

Review Stage at time of this submission: Completed but not published.

Conflicts of interest: None declared.

\section{A meta-analysis of high mobility group 1 frame protein levels in cerebrospinal fluid and serum of patients with epilepsy}

\author{
Chen, Y1; Li, HP2; Chen, XL33.
}

Review question / Objective: Inflammation plays a key role in the pathogenesis and progression of epilepsy. High mobility group frame protein 1 (HMGB1) is an important proinflammatory factor. The association between HMGB1 and epilepsy is controversial due to inconsistencies in experimental data. The purpose of this study was to quantitatively summarize and evaluate whether HMGB1 levels are associated with epilepsy.

Condition being studied: The pathophysiology of epilepsy is not completely clear, and nearly $30 \%$ of patients with epilepsy are still not satisfactorily controlled after drug treatment. There is increasing recognition of the need to identify individuals at risk for epilepsy and develop new approaches for prevention or treatment. Therefore, it is particularly important to excavate ideal biomarkers. Available evidence supports the role of pro-inflammatory cytokines in epileptic seizure mechanisms. HMGB1 is an important inflammatory mediator and has excellent theoretical potential as a biomarker and therapeutic indicator of epilepsy.

INPLASY registration number: This protocol was registered with the International Platform of Registered Systematic Review and Meta-Analysis Protocols (INPLASY) on 04 December 2021 and was last updated on 04 December 2021 (registration number INPLASY2021120029).

\section{INTRODUCTION}

Review question / Objective: Inflammation plays a key role in the pathogenesis and progression of epilepsy. High mobility group frame protein 1 (HMGB1) is an important pro-inflammatory factor. The association between HMGB1 and epilepsy is controversial due to inconsistencies in experimental data. The purpose of this study was to quantitatively summarize and evaluate whether HMGB1 levels are associated with epilepsy.

Condition being studied: The pathophysiology of epilepsy is not 
completely clear, and nearly $30 \%$ of patients with epilepsy are still not satisfactorily controlled after drug treatment. There is increasing recognition of the need to identify individuals at risk for epilepsy and develop new approaches for prevention or treatment. Therefore, it is particularly important to excavate ideal biomarkers. Available evidence supports the role of pro-inflammatory cytokines in epileptic seizure mechanisms. HMGB1 is an important inflammatory mediator and has excellent theoretical potential as a biomarker and therapeutic indicator of epilepsy.

\section{METHODS}

Search strategy: ("Epilepsy"[Title/Abstract] OR "Epilepsies"[Title/Abstract] OR "seizure disorder"[Title/Abstract] OR "seizure disorders"[Title/Abstract] OR "awakening epilepsy"[Title/Abstract] OR ( ( "epilepsie "[AII Fields] OR "Epilepsy"[MeSH Terms] OR "Epilepsy"[AII Fields] OR "Epilepsies"[All Fields] OR "epileps ys "[AII Fie Ids ]) AND "Awakening"[Title/Abstract]) OR "epilepsy cryptogenic "[Title/Abstract] OR "cryptogenic epilepsies"[Title/Abstract] OR "cryptogenic epilepsy"[Title/Abstract] OR "epilepsies cryptogenic"[Title/Abstract] OR "Aura"[Title/Abstract] OR "Auras"[Title/ Abstract]) AND ("hmgb1 protein"[Title/ Abstract] OR "HMG1"[Title/Abstract] OR ("FM1"[AII Fields] AND "gene product"[Title/Abstract]) OR "hmg 1 protein"[Title/Abstract] OR "box protein 1 high mobility group"[Title/Abstract] OR "Amphoterin "[Title/Abstract] OR "HMGB1"[Title/Abstract] OR "hmg 1 protein"[Title/Abstract] OR "heparin binding protein p30"[Title/Abstract] OR "heparin binding protein p30"[Title/ Abstract] OR ("p30"[All Fields] AND "heparin binding protein"[Title/Abstract])).

Participant or population: Epilepsy patients.

Intervention: HMGB1 levels in cerebrospinal fluid and serum.

Comparator: Healthy controls.
Study designs to be included: Case-control or cohort study.

Eligibility criteria: (1) Case-control study or cohort study design; (2) The original study reported data on cerebrospinal fluid or serum HMGB1 levels in controls and epileptic patients. Exclusion criteria: (1) Repeated studies using the same population or overlapping databases; (2) Non-human research; (3) Case reports, systematic evaluations, reviews, letters and reviews.

Information sources: PubMed, Web of Science, Embase, Cochrane library.

Main outcome(s): Name of first author, year of publication, test method, sample type, total number of cases, total number of controls, age, sex, HMGB1 concentration.

Quality assessment / Risk of bias analysis: Study selection was completed independently by two investigators according to pre-determined inclusion criteria, and disputes were resolved through discussion. The included studies were evaluated by two independent researchers using the Newcastle-Ottawa Scale. Studies that score $\geq 6$ are considered high quality.

Strategy of data synthesis: Statistical analysis was performed using Review Manager 5.3 and Stata 15.Mean standard deviation (SMD) and $95 \%$ confidence interval (CI) were used to analyze continuous variables (HMGB1 concentration). Cochrane $Q$ test and 12 statistics were used to assess inter-study heterogeneity. If the effect size of $Q$ test was $\mathrm{P}>0.05$ or $12<50 \%$, it was considered low heterogeneity. $Q$ test with $\mathbf{P}<\mathbf{0 . 0 5}$ or 12 of $50 \%$ to $75 \%$ is considered moderately heterogeneous; $12>75 \%$ is considered to be highly heterogeneous. If $P>0.05$ of $Q$ test or $12<50 \%$, fixed effects model was selected to evaluate merging SMD; otherwise, random effects model was used.

Subgroup analysis: Subgroup analysis by sample source, epilepsy type and patient age. 
Sensitivity analysis: Sensitivity analysis was performed using a "leave-one-out" method to verify whether the results of a metaanalysis are influenced by an individual study.

Country(ies) involved: China.

Keywords: Epilepsy; HMGB 1; MetaAnalysis.

Contributions of each author:

Author 1 - Yue Chen.

Author 2 - Huiping Li.

Author 3 - Xilu Chen. 